\title{
Shock Waves in the Solar Atmosphere
}

\author{
Fisenko, M. I. \\ UAFO, FEB RAS 692533, Russia
}

\begin{abstract}
The discovery of shock waves at observations sun like stars quasi-zero method. Shock waves associated with solar flares in Ha, it is shown that the amplitude of shock waves decreases to the edge of the sun, it follows that these waves are global. The conclusion is that the appearance of these waves may be connected with explosions in the deeper layers of the sun.
\end{abstract}

Key words: Shock waves, solar flares, the global wave of explosions in the deep layers of the sun.

\section{Introduction}

Solar flares are the most energetic events in the solar system, but compared to the total power of the sun they are barely noticeable. The authors of Ref. [1], it is shown that the total energy emitted by the flash is more than two orders of magnitude the amount of energy flash emitted in soft x-ray region. Flash gives a great contribution in the visible region of the solar spectrum. These results have implications for our understanding of solar-flare activity and variability of our star. They also showed that the bursts occur on a background of acoustic waves in the photosphere and continuous fluctuations in the granulation. Over a long period solar flux was considered constant, and only recently satellite experiments revealed that it varies continuously. To change in the solar flux is influenced by sub-surface convection in the sun and eruptive events. At very short time intervals of oscillations with a period of $300 \mathrm{sec}$. give to the flow of the sun $0.003 \%$ and the strongest solar flares to $0.015 \%$ [2]. On this basis, in our opinion, advisable to try to search for such oscillations in the global flow of sun-like stars, and possible connection of the oscillations with the solar activity phenomena.

\section{Materials and Methods}

Flow measurement of the sun carried out on

Corresponding author: Fisenko, M. I., researcher, research field: astrophysics. horizontal solar telescope ATSU-5 in the parallel beam in the period from 1980 to 1984 , the wavelength of maximum sensitivity of the radiation detector of 1.6 $\mu \mathrm{m}$. The measurements were used to zero (compensation) method, from the signal subtracted DC component, and the remainder increased.

Infrared radiation of the sun occurs under conditions of thermodynamic equilibrium and is characterized by the true kinetic temperature. It is formed in different layers of the solar atmosphere from the deepest observed at $1.6 \mu \mathrm{m}$ parts of the photosphere to the level of the temperature minimum in the chromosphere, observed in the millimeter region. Due to the limited spatial resolution of many infrared measurements as a good estimate in $\lambda(\mu=1) \mathrm{F}$ $\lambda$ is often taken as the value of $\mathrm{R} \lambda(\mu)$ on the surface of the disk changes little because of the smallness of the gradient $\partial \mathrm{T}(\tau \lambda=1) / \partial \lambda$. B $\lambda(\mu=1)$ is monochromatic brightness at the center of the disk, $\mathrm{F}$ $\lambda$ is the total solar flux and the relative change in brightness from center to $\operatorname{limb} \mathrm{R} \lambda(\mu)=\mathrm{B} \lambda(\mu) / \mathrm{B} \lambda(1)$ where $\mu=\cos \theta$ [3]. We note that the measurements were used the compensation method.

The observations were carried out in a broad band in the region of wavelengths over $1 \mu \mathrm{m}$. Calibration was conducted at the center of the sun in the focus of Newton grey wedge and a set of calibrated diaphragms.

The absolute flux was determined from the known energy distribution in the continuous spectrum of the 
sun according to the Labsa and Nickel [4]. As a registered device, it can be used as a compensation recorder G1 B1. The nonlinearity of $0.2 \%$, the deviation of the measuring ranges $1 \%$, the constancy of the zero point $0.1 \%$. In times of work of the system during 3 hours the change of zerolevel is certain, he was $1 \%$, and the drift of zero made a size $5 \times 10^{-5}$ $\mathrm{mV} / \mathrm{min}$ or $9.26 \times 10^{-7} \mathrm{mV} / \mathrm{sec}$. Standard compensation recorder operates on the principle of the compensator current or strain relief with continuous automatic adjustment.

\section{Resalts and Discussion}

The following figure shows the shock wave (Fig. 1).
Observations were made on 16 may 1984 with filter IKS-5. Fig. 1 shows world time UT. According to chromospheric observations in $\mathrm{N}$ a in the active region in $00 \mathrm{~h} 09 \mathrm{~m}$ observed two bright points with a jumper, connecting points. Most likely, it was a loop. The shock wave does not decay to $00 \mathrm{~h} 38 \mathrm{~m}$. In $00 \mathrm{~h} 36 \mathrm{~m}$ in the same active region began to flash as two bright dots, the jumper this time disappears. The maximum flow in the wave of $0.375 \mathrm{~mW} / \mathrm{cm} 2 \mu \mathrm{m}$, or $1.3 \times 10-4$ flux of the sun, or $5.02 \times 1029 \mathrm{erg} / \mathrm{sec}$. The shock wave we associate with a loop in the active region.

The following figure (Fig. 2) shows the shock wave that precedes a solar flare.

As shown in Fig. 2, the outbreak began in $02^{\mathrm{h}} 19^{\mathrm{m}}$,

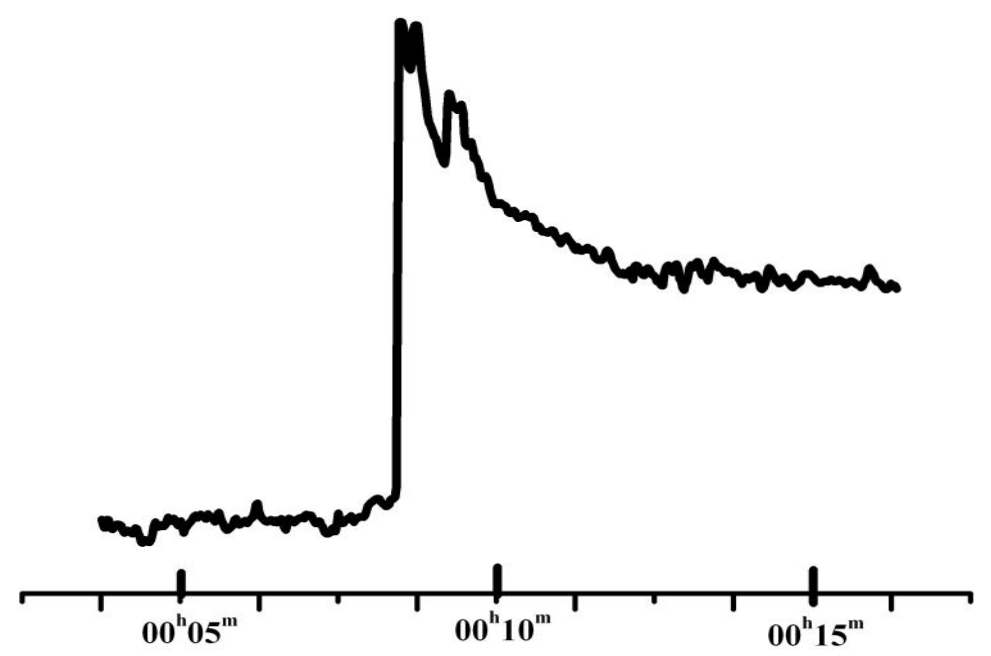

Fig. 1 Shock wave observed on 16 May 1984.

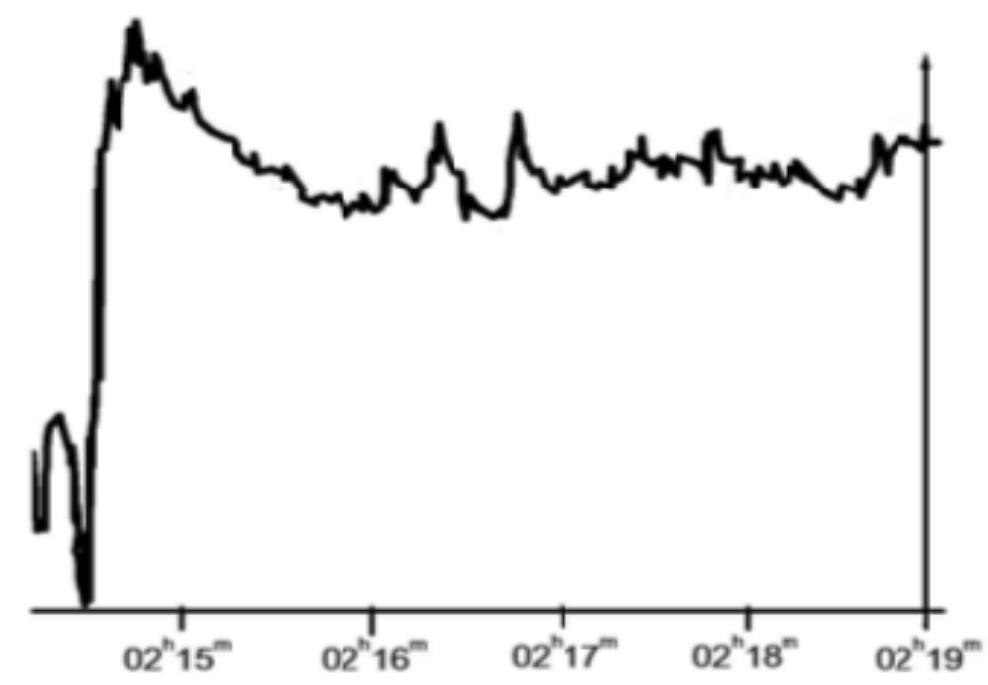

Fig. 2 Shock wave observed on 2 Feb. 1982. 


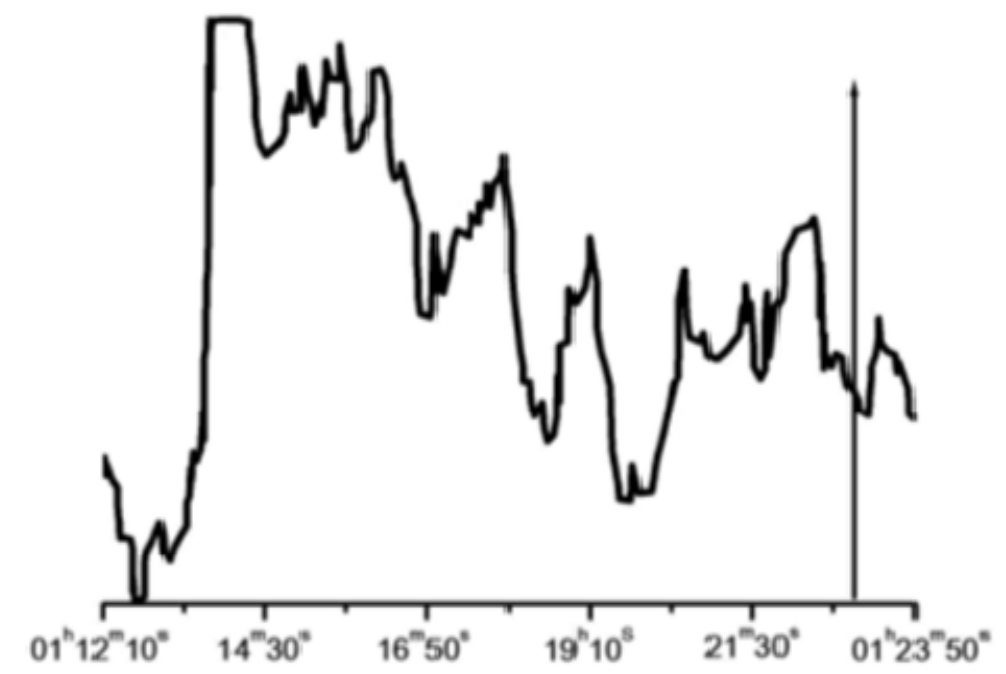

Fig. 3 The circumstances of a solar flare on 18 June 1982.

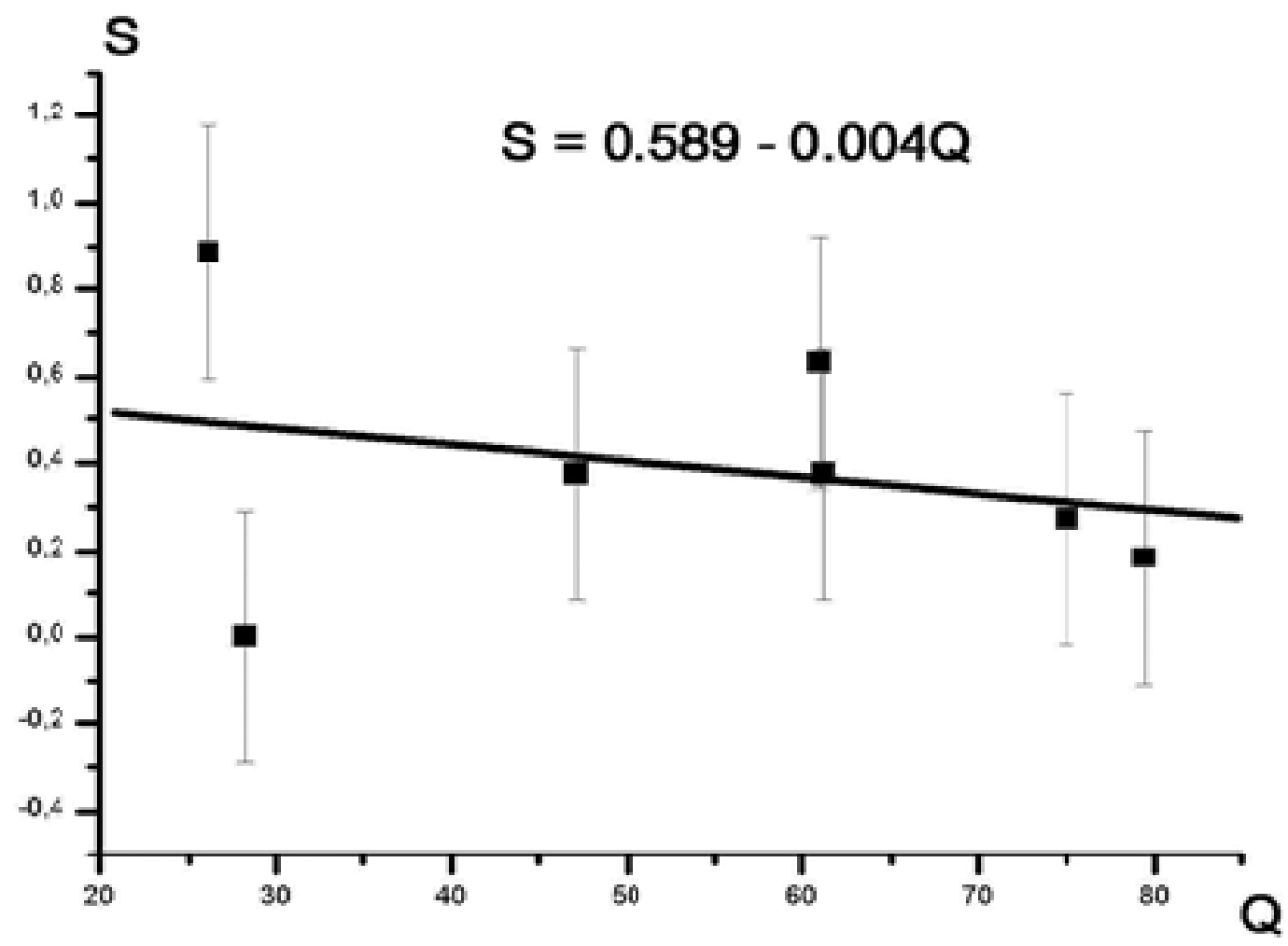

Fig. 4 The dependence of the maximum amplitude of the wave from the longitude.

as according VORO and according CULG. High according VORO $-02^{\mathrm{h}} 23^{\mathrm{m}}$, end $02^{\mathrm{h}} 27^{\mathrm{m}}$, according to CULG $-02^{\mathrm{h}} 24^{\mathrm{m}}, 02^{\mathrm{h}} 33^{\mathrm{m}}$, respectively. Coordinates S05E61, score $1 \mathrm{~F}$ according VORO. In the figure the beginning of the flare is shown by a vertical bar on $02 \mathrm{~h} 19 \mathrm{~m}$. Maximum flow in the wave $0.633 \mathrm{~mW} / \mathrm{cm} 2$ $\mu \mathrm{m}$ or $1.6 \times 10-3$ or $6.2 \times 1030 \mathrm{erg} / \mathrm{sec}$.
The following figure (Fig. 3) shows the circumstances of a solar flare on 18 June 1982. Beginning flash at $01^{\mathrm{h}} 23^{\mathrm{m}}$, high $01^{\mathrm{h}} 34^{\mathrm{m}}$, the end $02^{\mathrm{h}}$ $26^{\mathrm{m}}$. Coordinates N14 E75, score $1 \mathrm{~N}$. The maximum amplitude of the shock wave is $0.273 \mathrm{~mW} / \mathrm{cm}^{2} \mu \mathrm{m}$ or $9.5 \times 10^{-4}$, or $3.67 \times 10^{30} \mathrm{erg} / \mathrm{sec}$. Wave duration of $393 \mathrm{sec}$. 


\section{Conclusions}

We found only 7 of the shock waves. The following figure (Fig. 4) shows the dependence of the maximum amplitude of the wave from the longitude of $\mathrm{Q}$.

Fig. 4 shows the decrease in the amplitude of the shock waves in front of the flash to the edge of the Sun. One axis shows the maximum amplitude $\mathrm{S}$, and the second the longitude of Q. the Amplitude of shock waves decreases towards the edge, i.e. the contribution to the amplitude of optically thin layers is small, therefore, these waves can be global. Perhaps they are associated with explosions in the deeper layers of the sun [5].

\section{References}

[1] Kretzschmar, M., Dudok de Wit, T., Schmutz, W., Sabri, M., and Jean, H. 2010. "The Effect of Flares on Total Solar Irradiance.” Nature Physics 6: 690-2.

[2] Fröhlich, C., and Lean, J. "Solar Radiative Output and Its Variability: Evidend and Mechanisms." [Electronic resource] http://www.rivernet.nsu.edu/courselocker /PaleoClimate/FrohlichLeanSolIrdoverviev.pdf.

[3] Lena, P. 1979. "Infrared Observations of the Sun." Infrared and Submillimeter Astronomy. M.: Mir, P., 106-17.

[4] White, A. 1980. Flow of Energy of the Sun and Its Changes. Moscow: Mir, C., 558.

[5] Stanislav Kozlov. Analytical. Physics [Electronic resource]. http://ru.newastrophysics.com/wp/ category/articles. 